\title{
Technical Refinement of the Orage Ga-FIB column and Optimizing its Control for Routine Analytical Tasks
}

Tomáš Hrnčíŕ ${ }^{1}$, Miroslav Rudolf ${ }^{1}$, Mikuláš Kocman ${ }^{1}$, Jean-Baptiste Mellier ${ }^{2}$, Laurent Alcaraz ${ }^{2}$ and Anne Delobbe $^{2}$

1. TESCAN Brno s.r.o., R\&D Physics Team, Brno, Czech Republic

2. Orsay Physics S.A., Fuveau, France

The new TESCAN S8000G FIB-SEM instrument is now equipped with the newly-introduced Orage FIB, a novel Ga-FIB column from Orsay Physics [1]. The next generation Ga-source FIB column with cuttingedge ion optics delivers ultra-fine resolution throughout the entire range of beam energies from $500 \mathrm{eV}$ to $30 \mathrm{keV}$, as well as excellent high-current performance at $30 \mathrm{keV}$.

The Orage column achieves ion-beam currents up to 100 nA (measured on the sample stage Faraday cup) enabling fast sputtering rates for enhanced volume-wise analytical capabilities including fast preparation of large-area cross-sections and rapid FIB-tomography. The spot shape is well defined even at $100 \mathrm{nA}$; this goal was achieved by combining optimization of FIB optics and ultra-precise piezo-driven gun centering that refines manual gun alignment.

The performance of the Orage column is improved over the whole ion-beam energy range compared to the previous Cobra column. For example, it enhances accurate, site-specific and damage-free TEM sample preparation at beam energies of $1-2 \mathrm{keV}$, thus minimizing the amorphous damage caused by Ga FIB milling [2]. Beam energy as low as $500 \mathrm{eV}$ is also possible, although it does not offer any advantage for typical TEM sample cleaning, probably because the sputter yield can drop below 1 and more Ga can be deposited on the sample [2].

Our recent experiments on piezo gun centering have shown excellent repeatability of the order of $\sim 100 \mathrm{~nm}$ - an excellent result given the weight of the movable gun parts (several $\mathrm{kg}$ ). Extensive tests were performed, simulating the load applied to gun centering during the lifetime of the FIB column, with negligible effects on the repeatability of the centering.

Piezo-driven apertures with a similar repeatability were installed enabling precise, rapid and fullyautomated mechanical alignment with high reproducibility over the whole beam-energy range. This allows optimum mechanical alignment of the column, where the best FIB resolution or the optimum spot shape for milling is guaranteed. The number of available beam-limiting apertures in the aperture strip was increased to 30 and its configuration has been optimized to obtain longer lifetime of the aperture strip under routine operating conditions.

A significant improvement in the stability of both beam current and FIB presets in general was brought about by an automated ion-source heating routine together with constant extractor voltage operation during the lifetime of the ion source. This is also highly advantageous for applications requiring long FIB milling times like multiple TEM sample preparation, FIB-SEM 3D tomography and particularly 3D EDX and EBSD, where the beam has to be stable for up to several days during continuous Ga-source consumption. 
The semi-automatic spot shape tuning wizard helps the FIB operator to obtain the best shape of FIB spots on the sample, i.e. to optimally combine parameters like beam aperture centering, stigmation octupole settings and objective lens focus - see Figure 1. The corresponding values are automatically saved as a user preset. This preset can then be recalled automatically and usually no further adjustments are necessary.

FIB optical parameters like spot size or probe current are calculated continuously during FIB column usage, depending on the beam energy, lens voltage or aperture size. They help the user to tune the FIB for specific applications, like e.g. optimized milling or deposition rate.

References:

[1] T. Hrnčír et al., EUFN Workshop Proceedings (2017), 37.

[2] L. A. Giannuzzi, B. Van Leer, and J. Ringnalda, Microsc Microanal 13(Suppl 2) (2007), 1516.

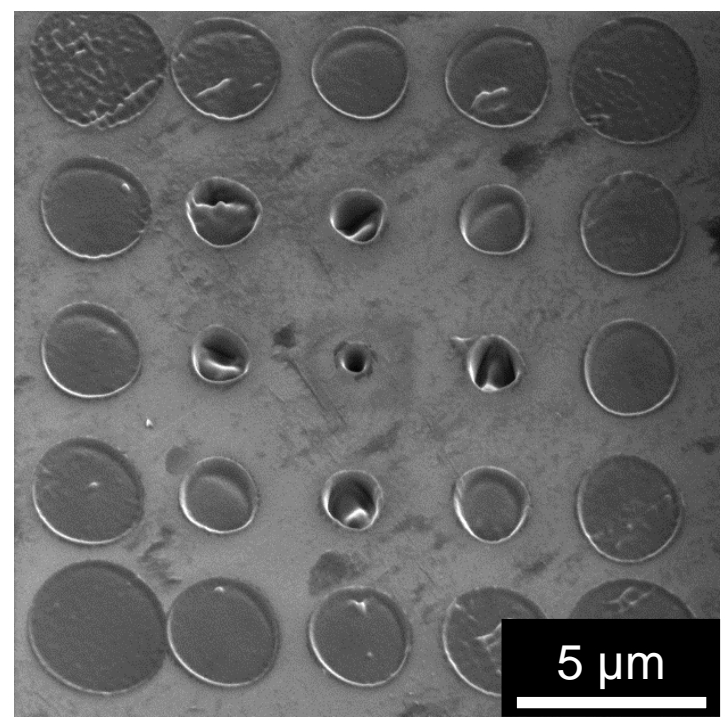

Figure 1. Semi-automated tuning of the FIB spot on the sample for a polishing preset at a beam current of $1 \mathrm{nA}$ and a beam energy of $30 \mathrm{keV}$. The milling time for each spot is $3 \mathrm{~s}$ and the stigmation octupole voltages are changed in two perpendicular directions to find the optimum stigmation adjustment under the given conditions. A similar matrix is also automatically generated for aperture centering and objective lens voltage to obtain column parameters for the best spot shape at a given beam energy and probe current. 